\title{
Efecto de la manipulación del semen criopreservado de bovinos Bos Taurus sobre la integridad espermática
}

\section{Bos Taurus cattle sperm integrity effect by cryopreserved semen manipulation}

Fecha recepción: 22 de junio de 2015

Fecha aceptación: 23 de diciembre de 2015

\author{
Norberto Villa-Duque ${ }^{1}$ \\ Claudia Marcela Amaya-Torres ${ }^{2}$ \\ Darwin García-Rojas ${ }^{3}$ \\ Natalia Nieto-Omeara ${ }^{4}$ \\ Natalia Terán-Acuña ${ }^{5}$
}

\section{Resumen}

En el estudio se evaluó el efecto de descongelar y aplicar semen de bovinos Bos Taurus en 33 ganaderías del Magdalena Medio colombiano, y se estudió in vitro el efecto de la injuria encontrada sobre la integridad de las membranas espermáticas. La información en fincas se recopiló mediante formulario específico, mientras que el estudio in vitro se ejecutó en el laboratorio de Biotecnología Reproductiva Animal del Instituto Universitario de la Paz (Barrancabermeja, Santander). El estudio consistió en someter pajillas comerciales de $0.5 \mathrm{ml}$ de toros Holstein y Pardo Suizo a la técnica convencional y a tres modificaciones de esta (injurias) mediante un diseño randomizado. Ninguna de las fincas evaluadas aplicó correctamente la práctica de la inseminación artificial; errores notorios fueron: exceso de tiempo durante la extracción de la pajilla, descongelación en la región axilar y no combinación correcta entre tiempo y temperatura. Los resultados evidenciaron diferencia significativa $(\mathrm{P}<0.05)$ por efecto de la raza para la integridad y resistencia de las membranas espermáticas, para la integridad de las membranas por efecto de los tratamientos cuando la pajilla se descongeló a temperatura corporal en la región axilar y para la integridad de la membrana acrosomal cuando la extracción de la pajilla se realizó en forma incorrecta. El semen de la raza Holstein evidencia una ligera tendencia a ser más resistente que el de la raza Pardo Suizo.

Palabras clave: acrosoma; biotecnología reproductiva; genotipo; inseminación artificial; plasmática.

1 M. Sc. Instituto Universitario de la Paz (Barrancabermeja, Colombia).norberto.villa@unipaz.edu.co.

Profesional Independiente.

Instituto Universitario de la Paz (Barrancabermeja, Colombia).

Instituto Universitario de la Paz (Barrancabermeja, Colombia).

Instituto Universitario de la Paz (Barrancabermeja, Colombia). 


\begin{abstract}
This study evaluated the semen thawing and application effect in 33 bovine cattle farms in the Colombian Magdalena Medio region and studied in vitro, the injury effect found on the integrity of sperm membranes. The information was collected in farms by a specific paper form. The in vitro study was carried out in the Reproductive Animal Biotechnology laboratory at the Instituto Universitario de la Paz (Barrancabermeja, Santander, Colombia). The study consisted of exposing commercial $0.5 \mathrm{ml}$ straws of bull Holstein and Brown Swiss to the conventional technique, and three modifications thereof (injuries) by a randomized design. None of the farms evaluated applied correctly the practice of artificial insemination. Evident errors were: Time excess for extraction of the straw, thawing in the axillary region and incorrect combination of time and temperature. The results showed significant difference $(P<0.05)$ by the race effect for the integrity and strength of the plasma membrane, also for the integrity of the membranes by treatment effect, when the straw was thawed at body temperature in the axillary region and for the integrity of the acrosomal membrane when extraction straw was performed incorrectly. Holstein semen evidences a slight tendency to be more resistant than the Brown Swiss breed.
\end{abstract}

Keywords: acrosome; artificial insemination; genotype; plasma; reproductive biotechnology. 


\section{Introducción}

La inseminación artificial (IA) es la biotecnología reproductiva más empleada en las empresas ganaderas; su consolidación se ha logrado a partir del uso de semen congelado empacado en pajillas de 0.25 y $0.5 \mathrm{ml}$, el cual es aplicado en el cuerpo del útero mediante una técnica instrumental (TI) específica (1), y su utilización ha contribuido notoriamente al mejoramiento genético $y$, consecuencialmente, al aumento de la productividad; no obstante, fallas en la práctica instrumental pueden afectar de manera grave la célula espermática, en detrimento de la fertilidad y la productividad $(2,3)$.

La evaluación de la fertilidad de un reproductor y de la manera como es afectada por la manipulación del semen, sea en fresco o criopreservado, requiere mayor estudio de los factores involucrados en el proceso $(4,5)$. La calidad del semen de un toro puede afectarse intrínseca y extrínsecamente (4); en el primer caso, puede ser por factores genéticos, nutricionales, tóxicos, de estrés y edad (6), mientras que en el segundo, por fallas en su manipulación, derivadas de los protocolos de congelación y descongelación o de la no permanencia a las temperaturas indicadas $(7,8)$.

La criopreservación de semen a $-196{ }^{\circ} \mathrm{C}$ detiene todos los procesos metabólicos, lo que permite su conservación de manera indefinida (9); sin embargo, tanto en la criopreservación como en la descongelación se pueden presentar errores que inducen alteraciones en las membranas celulares, los organelos y la interacción célula-célula, que a la postre afectan la capacidad fecundante del espermatozoide (10). El retorno a las condiciones fisiológicas, el cual ocurre durante la descongelación, es crítico para la supervivencia celular (11), por lo que exige máximo cuidado con el tiempo, la temperatura y la manipulación de la pajilla; así, por ejemplo, la descongelación de pajillas durante más de 30 segundos en temperaturas mayores a los $37{ }^{\circ} \mathrm{C}$ redundará en mayor número de alteraciones espermáticas $(7,12)$, y en porcentajes más altos de retorno al celo $(3,13)$. Alteraciones similares suceden cuando se manipulan las pajillas por fuera del cuello del termo de almacenamiento, o cuando los niveles de nitrógeno líquido son insuficientes para mantener la temperatura de $-196{ }^{\circ} \mathrm{C}(14)$. Además, las bajas temperaturas están asociadas con el aumento de la rigidez de la membrana espermática y con la reducción de la velocidad del movimiento de moléculas a través de la membrana, mediante los procesos de transporte activo, que terminan sometiendo la célula a estrés osmótico $(15,16)$ o a shock por frío, con los consecuentes cambios en la estructura del acrosoma $(2,17)$.

Con el objetivo de detectar errores de los inseminadores en la implementación de la TI con semen congelado, que conllevan injuria a la célula espermática, se observaron 33 hatos en el Magdalena Medio colombiano, se replicaron los principales de ellos en el Laboratorio y se evaluó el efecto de tales errores sobre la integridad de las membranas espermáticas.

\section{Materiales y métodos}

Primera fase: A través de la observación y mediante el diligenciamiento de un formato, se detectaron los principales errores que cometieron 33 inseminadores al implementar la $\mathrm{TI}$ en hatos con programas de IA; esto permitió obtener información acerca de las características críticas del proceso. Los factores contemplados en el formato fueron: estado físico del termo, su sitio de almacenamiento, el nivel del nitrógeno líquido, la temperatura y el tiempo durante las diferentes etapas del proceso, la forma de extracción, descongelación y puesta de la pajilla en el cuerpo del útero.

Segunda fase: Mediante revisión bibliográfica, se seleccionaron los errores que mayor injuria le podrían causar a la integridad de la célula espermática, y se implementó un modelo experimental in vitro, en el Laboratorio de Biotecnología Reproductiva Animal (LABRA) del Instituto Universitario de la Paz (Barrancabermeja, Santander, Colombia). Se establecieron 4 tratamientos, 2 razas del Bos Taurus por tratamiento (Holstein y Pardo Suizo) y 3 réplicas por raza. Se utilizaron pajillas comerciales

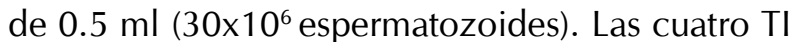
diseñadas fueron: TI 1. Protocolo convencional, es decir, el protocolo establecido: extraer la pajilla en un tiempo no superior a 15 segundos y a nivel del 
cuello del termo y descongelarla en baño María a $37^{\circ} \mathrm{C}$ durante 30 segundos. TI 2. Descongelación de las pajillas en la región axilar, es decir, por el calor corporal en la parte ventral de la región axilar, durante 30 segundos. TI 3. Descongelación de la pajilla durante ocho minutos, en agua a $37{ }^{\circ} \mathrm{C}$. TI 4. Extracción incorrecta de la pajilla; en esta técnica, la canastilla y la escalerilla con las pajillas fueron expuestas por fuera del cuello del termo de criopreservación, durante 30 segundos, tres veces al día, cuatro días consecutivos, antes de utilizar su contenido para el análisis in vitro. Con el fin de descartar el criopreservante, el cual imposibilita la evaluación objetiva de las variables, una vez realizada la descongelación de la pajilla en los cuatro protocolos, se diluyó el semen en 3.5 $\mathrm{ml}$ de solución salina fosfato-bufferada (PBS), a 37 ${ }^{0} \mathrm{C}$, se centrifugó a 500 g durante 5 minutos y se eliminó el sobrenadante; este proceso se realizó dos veces, y el sedimento se utilizó para realizar el análisis in vitro $(18,19)$. Teniendo en cuenta que la centrifugación acentúa algunas alteraciones de la célula espermática, en este experimento todas las réplicas fueron sometidas al mismo tratamiento; es de anotar que, no obstante, (20) encontró alteraciones de la membrana plasmática solo cuando centrifugó el semen en peróxido de hidrógeno a $700 \mathrm{~g}$ y durante 45 minutos. Las variables evaluadas fueron: Integridad de la Membrana plasmática (IMp), cuya valoración se hizo mediante la prueba Hipoosmotic Swelling Test (HOST) (21); Resistencia de la Membrana plasmática (RMp), utilizando la prueba Osmotic Resistance Test (ORT) (21), e
Integridad de la Membrana acrosomal (IMa), cuya evaluación se realizó siguiendo el protocolo propuesto por $(22,23,24)$. Para los conteos se utilizó el microscopio de contraste de fase (magnificación 400X). Los resultados se expresaron en porcentaje de espermatozoides positivos (para IMp y RMp) y con cresta apical normal con dos conteos de 100 (para IMa). RMp/IMa, la resistencia de la membrana plasmática y la integridad de la membrana acrosomal, se realizó fijando los espermatozoides con glutaraldehido al $2 \%$ en una solución ORT (Osmotic Resistance Test, en dilución 1:1), utilizando un Microscopio de contraste de fases (magnificación 400X) (25). El resultado se dio en porcentaje.

Para el análisis de los resultados de la primera fase se utilizó la estadística descriptiva, y para la segunda fase se usaron gráficos de cajas y bigotes "BOXPLOT" y tablas de contingencia. Se compararon los resultados en cada raza respecto a los tratamientos usados mediante ANOVA. Para las comparaciones múltiples se utilizó TUKEY. Las pruebas estadísticas se compararon al nivel de significación máxima de 0.05 , usando el programa IBM SPSS Statistics 22.0.

\section{Resultados y discusión}

Primera fase: Los hallazgos encontrados en las 33 fincas (Tabla I) evidencian irregularidades en todas las características observadas, las cuales comprometen la viabilidad espermática y, consecuencialmente, la fertilidad del hato (26).

Tabla I. Síntesis de los hallazgos para las variables evaluadas.

\begin{tabular}{|l|c|c|}
\hline \multicolumn{1}{|c|}{ Ítem } & Con fallas (\%) & Sin fallas (\%) \\
\hline Condiciones del termo & 3 & 97 \\
\hline Almacenamiento del termo & 85 & 15 \\
\hline Nivel de nitrógeno & 18 & 82 \\
\hline $\begin{array}{l}\text { Extracción y descongelación del semen (extrac- } \\
\text { ción incorrecta de la pajilla- descongelación en } \\
\text { la región axilar- descongelación por más de 30 } \\
\text { segundos) }\end{array}$ & 95 & 5 \\
\hline Características de la inseminación & 90 & 10 \\
\hline
\end{tabular}


El promedio del tiempo utilizado para la extracción de la pajilla en las 33 fincas observadas estuvo por encima del máximo permitido (10-15s); en varios casos se observó la manipulación de la pajilla por fuera del cuello del termo, esto produce cambios de temperatura en la pajilla, lo que conlleva alteraciones de los organelos de la célula espermática -el daño producido es proporcional al grado y duración del calentamiento, no se puede determinar externamente, ni se arregla al almacenar la pajilla otra vez correctamente $(27,28)$; a pesar de que la pajilla debe ser utilizada en los primeros cinco minutos después de su descongelamiento, varios de los inseminadores observados demoraron más de 5 minutos, situación que compromete la membrana espermática, pues al ser sometida a estrés ocasiona en ella cambios lipídicos de difícil reversión $(16,29)$; aunque el protocolo convencional recomienda descongelar la pajilla a $37^{\circ} \mathrm{C}$ durante 30 segundos, en un baño María (3), se observaron inseminadores descongelándola en la región axilar.

Segunda fase: En los resultados, de la Tabla II, se puede evidenciar que existe diferencia significativa $(\mathrm{P}<0.05)$ por efecto de la raza para la integridad y resistencia de las membranas espermáticas.

Tabla II. Efecto de la raza sobre la integridad de las membranas espermáticas.

\begin{tabular}{|l|l|l|l|l|}
\hline Genotipos & IMp & RMp & IMa & IMp/IMa \\
\hline Holstein & 0,015 & 0,001 & 0,001 & 0,002 \\
\hline Pardo Suizo & 0,001 & 0,001 & 0,001 & 0,001 \\
\hline
\end{tabular}

Tabla III. Comparación de la integridad de la membrana plasmática (IMp) entre tratamientos por raza mediante Tukey.

\begin{tabular}{|c|c|c|c|c|c|c|}
\hline \multirow{2}{*}{ Tratamientos } & \multicolumn{3}{|c|}{ Holstein } & \multicolumn{3}{c|}{ Pardo Suizo } \\
\cline { 2 - 7 } & $\mathrm{T} 1$ & $\mathrm{~T} 2$ & $\mathrm{~T} 3$ & $\mathrm{~T} 1$ & $\mathrm{~T} 2$ & $\mathrm{aa}$ \\
\hline $\mathrm{T} 4$ & $\mathrm{aa}$ & $\mathrm{aa}$ & $\mathrm{aa}$ & $\mathrm{ab}$ & $\mathrm{aa}$ & \\
\hline $\mathrm{T} 3$ & $\mathrm{ab}$ & $\mathrm{aa}$ & & $\mathrm{ab}$ & & aa \\
\hline $\mathrm{T} 2$ & $\mathrm{ab}$ & & $\mathrm{aa}$ & $\mathrm{ab}$ & & a \\
\hline
\end{tabular}

* Letras diferentes indican diferencia significativa $(p<0,05)$.

Tabla IV. Comparación para la resistencia de la membrana plasmática (RMp), la integridad de la membrana acrosomal (IMa) y la RMp/IMa mediante Tukey.

\begin{tabular}{|c|c|c|c|c|c|c|}
\hline \multirow{2}{*}{ Tratamientos } & \multicolumn{3}{|c|}{ Holstein } & \multicolumn{3}{c|}{ Pardo Suizo } \\
\cline { 2 - 7 } & $\mathrm{T} 1$ & $\mathrm{~T} 2$ & $\mathrm{~T} 3$ & $\mathrm{~T} 1$ & $\mathrm{~T} 2$ & $\mathrm{~T} 3$ \\
\hline $\mathrm{T} 4$ & $\mathrm{ab}$ & $\mathrm{aa}$ & $\mathrm{aa}$ & $\mathrm{ab}$ & $\mathrm{aa}$ & $\mathrm{aa}$ \\
\hline $\mathrm{T} 3$ & $\mathrm{ab}$ & $\mathrm{aa}$ & & $\mathrm{ab}$ & $\mathrm{aa}$ & \\
\hline $\mathrm{T} 2$ & $\mathrm{ab}$ & & $\mathrm{aa}$ & $\mathrm{ab}$ & & $\mathrm{aa}$ \\
\hline
\end{tabular}

* Letras diferentes indican diferencia significativa $(p<0,05)$.

Según (24), el semen contenido en las pajillas resiste de diferente manera los efectos detrimentales de la criopreservación sobre la integridad de las membranas espermáticas; lógicamente, y en ese mismo orden de ideas, resisten la injuria a la que son sometidas en el proceso de implementación de la TI en los programas de IA. Situación grave, si se tiene en cuenta que solo los espermatozoides que son capaces de realizar la reacción acrosomal de manera sincronizada con la fase de penetración del oocito, tienen la habilidad de pasar a través de la zona pelúcida y, como consecuencia, fusionarse con este para formar un embrión (30). En las investigaciones de (31), realizadas en estas mismas razas, aunque las medias disminuyeron a causa de la manipulación (criopreservación), no hubo dife- 
rencia significativa $(p<0,05)$ para la integridad de las membranas espermáticas.

Los resultados de la Tabla $\mathrm{V}$ muestran diferencia significativa $(p<0,05)$ para la IMa, cuando se implementó el protocolo convencional (T1); diferen- cia significativa $(\mathrm{P}<0,05)$ para la IMp, la IMa y la RMp/IMa, cuando la pajilla se descongeló a temperatura corporal en la región axilar (T2), y para la IMa, cuando la extracción de la pajilla se realizó en forma incorrecta (T4).

Tabla V. Efecto de los tratamientos sobre la integridad espermática.

\begin{tabular}{|c|c|c|c|c|}
\hline \multirow{2}{*}{ Variable } & \multicolumn{4}{|c|}{ Sig. } \\
\cline { 2 - 5 } & $\mathrm{T} 1$ & $\mathrm{~T} 2$ & $\mathrm{~T} 3$ & $\mathrm{~T} 4$ \\
\hline $\mathrm{IMp}$ & 0,840 & 0,031 & 0,834 & 0,146 \\
\hline $\mathrm{RMp}$ & 0,135 & 0,064 & 0,295 & 0,344 \\
\hline $\mathrm{IMa}$ & 0,010 & 0,014 & 0,109 & 0,031 \\
\hline $\mathrm{RMp} / \mathrm{IMa}$ & 0,165 & 0,035 & 0,106 & 0,096 \\
\hline
\end{tabular}

Las afectaciones parten del envasado del semen; según (32), la diferencia más importante es la relación superficie-volumen, ya que determina la velocidad a la que se suceden los cambios de temperatura dentro de la dosis. Debido a que la relación superficie-volumen es amplia en la pajilla, la hace significativamente más sensible a la manipulación incorrecta en el momento de la extracción o cuando se tiene que pasar de un termo a otro, afectándose fundamentalmente la integridad de las membranas espermáticas. (33) afirma que la manipulación rutinaria de las canastillas, la cual

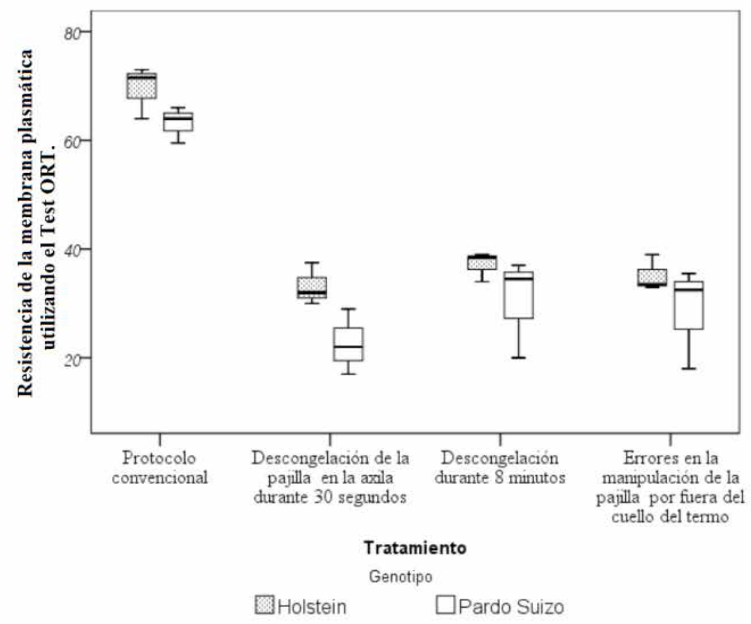

implica subirlas hasta el cuello del termo, afecta notoriamente los organelos de la célula espermática, debido a las fluctuaciones de temperatura a la que son sometidas constantemente. (34) simularon esta situación y encontraron que las pajillas, sobre todo las almacenadas en la parte superior del termo, sufrieron más daño acrosomal que las ubicadas en la parte inferior. Sin embargo, (35) no encontró variaciones en la integridad espermática trabajando de 6 a 18 meses en termos utilizados en programas de IA.

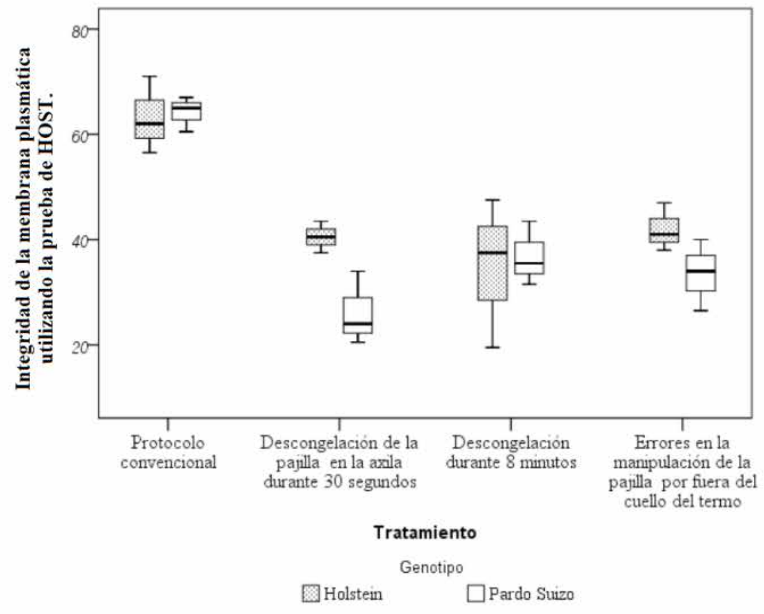

Figura 1. Comparación de medias para la resistencia de la membrana plasmática (RMP) y la integridad de la membrana plasmática (IMP). 
Respecto al genotipo, el trabajo de (36) reporta que el semen de la raza Holstein evidencia una tendencia a ser más resistente a la injuria que el de la raza Pardo Suizo, coincidiendo con los resultados de esta investigación. (37) afirma, en sus investigaciones con semen de toro Holstein, que los cambios que llevan a una calidad inaceptable para inseminar (según normas ISO 9002) se refirieron a demoras en retirar la pajilla del termo de nitrógeno, a cambios bruscos de temperatura de descongelado, así como a demoras en efectivizar la inseminación una vez descongelada la dosis.
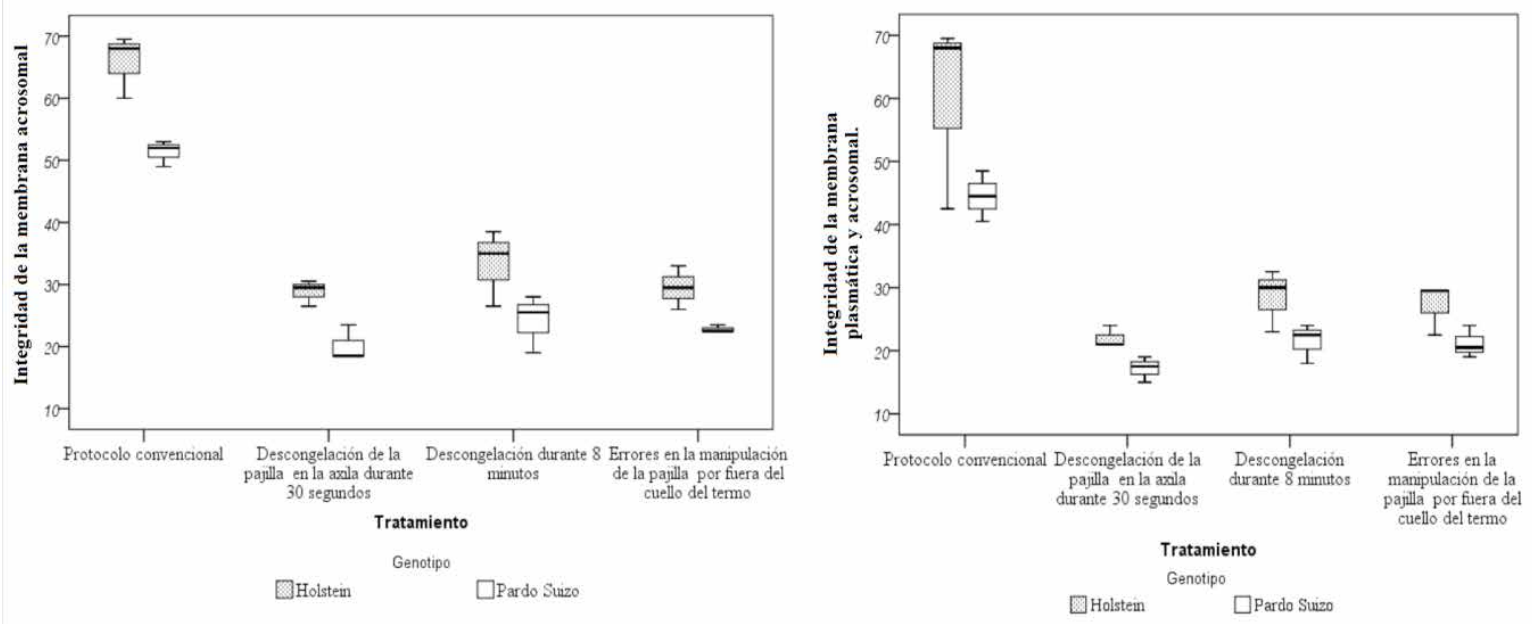

Figura 2. Comparación de medias para la integridad de la membrana acrosomal (IMa) y la integridad de la membrana plásmatica y acrosomal (IMp/IMa).

En investigaciones que evaluaron el efecto de la criopreservación sobre las variables trabajadas se evidencia el efecto de la criopreservación sobre ambas membranas (plasmática y acrosomal), con resultados porcentuales más altos $(41 \%$ para la IMp y $48 \%$ para IMa) que los obtenidos en este trabajo $(31,38)$, lo que estaría sugiriendo un daño agregado por las fallas en la manipulación de las pajillas al momento de implementar la TI en la IA. Los resultados en la evaluación de la IMp y de la IMa son muy relevantes debido a que el ORT ha demostrado tener una correlación alta y positiva con la capacidad fecundante del espermatozoide (38). Sin duda alguna, las fallas en la manipulación de la pajilla afectan la funcionalidad espermática causando un efecto deletéreo sobre la función del acrosoma $(39,40)$ y de la membrana plasmática, potencial de membrana mitocondrial e integridad del ADN (41). Los espermatozoides sometidos a injuria están sujetos a una serie de alteraciones en su estructura, tales como dilatación y rompimiento a nivel de la membrana, con cambios de permeabilidad en esta y cambios en la estructura de fosfolípidos y proteínas, que traen como consecuencia una reducción en su motilidad y viabilidad espermáticas $(42,43)$.

\section{Conclusiones}

En la implementación de la técnica instrumental en los programas de inseminación artificial analizados, las observaciones a los 33 inseminadores permitieron detectar fallas de gran relevancia para la calidad del semen congelado-descongelado. La integridad de las membranas espermáticas se vio afectada en cada uno de los genotipos evaluados cuando se implementaron variaciones en el protocolo convencional, mientras que los daños detrimentales se evidenciaron cuando las pajillas fueron descongeladas en la región axilar. El semen de la raza Holstein evidencia una ligera tendencia a ser más resistente que el de la raza Pardo Suizo. 


\section{Referencias}

(1) Foote RH. The history of artificial insemination: Selected notes and notables. Journal of Animal Science, 2002; 80: 1-10.

(2) Nur Z., Lleri I K., Ak K. Effects of different temperature treatments applied to deep stored bull semen on post-thaw cold shocked spermatozoa. Bulletin of Veterinary Institute Pulawy, 2006; 50(1): 79-83.

(3) Pace M M., Sullivan J J., Elliott F I., Graham E F., Coulter G H. Effects of thawing temperature, number of spermatozoa and spermatozoal quality on fertility of bovine spermatozoa packaged in $5 \mathrm{ml}$ french straws. Journal Animal Science, 1981; 53: 693-701.

(4) Barth AD, Waldner CL. Factors affecting breedings soundness classification of beef bulls examined at the Western college of Veterinay Medicine. Canadian Veterinary Journal, 2002; 43: 274-284.

(5) Saacke R. Fertilidad del toro: una opinión sobre su estado actual y perspectivas. Taurus, 2003; 5: 18-28.

(6) Spitzer J. Evaluación de la salud reproductiva del toro: estado actual. International Veterinary Information Service, 2000. Disponible en: www. ivis.org.

(7) Correa JR, Rodríguez MC, Patterson DJ, Zavos P. Thawing and proccesing of cryopreserved bovine spermatozoa at various temperatures and their effect on sperm viability, osmotic shock and sperm membrane functional integrity. Theriogenology, 1996, 46: 413-420. DOI: http://dx.doi.org/10.1016/0093-691X(96)00163-X.

(8) Pickett BW, Berndtson WE, Sullivan FT. Influence of seminal additives and packaging systems on fertility of frozen bovine spermatozoa. Journal of Animal Science, 1978; 47: 12-47.
(9) Nebel RL. Thechniques for Artificial Insemination of cattle whith frozen-thawed semen. R. S. Youngquist (ed.): Current therapy in large animal theriogenology, 1997; 1: 251-256.

(10) Woods E., Benson J., Agca Y., Critser J. Fundamental criobiology of reproductive cells and tissues. Criobiology, 2004; 48: 146156. DOI: http://dx.doi.org/10.1016/j.cryobiol.2004.03.002.

(11) Mazur P. Freezing of living cells:mechanisms and implications. American Journal of Phisiology, 1984; 247: 145-142.

(12) Rodríguez OL, Berdtson WE, Ennen BD, Pickett BW. Effect of rates of freezing, thawing and level of glycerol on the survival of bovine spermatozoa in straws. Journal Animal Science, 1975; 41: 129-136.

(13) Almquist JO, Grube KE, Rosenberger JL. Effect of thawing time on fertility of bovine spermatozoa in french straws. Journal Dairy Science, 1982; 65: 824-827. DOI: http://dx.doi. org/10.3168/jds.S0022-0302(82)82271-6.

(14) Berndtson WE, Pickett BW. Thecniques for the cryopreservation and fiel handling of bovine spermatozoa. N. A. o. Sciences, 1978.

(15) Mendoza JA, Dulin P., Warrant T. The Lower Hydrolyisis of ATP by the Stress Protein GroEL Is a Major Factor Responsible for the Diminished Chaperonin Activity at Low Temperature. Criobiology, 2000; 41: 319-323. DOI: http://dx.doi.org/10.1006/cryo.2000.2287.

(16) Watson PF. The causes of reduced fertility with cryopreserved semen. Animal Reproduction Science, 2000; 60: 481-492. DOI: http:// dx.doi.org/10.1016/S0378-4320(00)00099-3.

(17) Brown JL, Senger PL, Hillers JK. Influence of thawing time and post-thawing temperature on acrosomal maintenance and motility of bovine spermatozoa frozen in $0.5 \mathrm{ml}$ french straws. Journal Animal Science, 1982; 54: 938-944. 
(18) Brackett BG, Oliphant G. Capacitation of rabbit spermatozoa in vitro. Biology of Reproduction, 1975; 12: 260-274. DOI: http://dx.doi. org/10.1095/biolreprod12.2.260.

(19) Risopatron JR, Sánchez N., Sepúlveda E., Pe-a P. Selección de espermatozoides de bovino desde semen congelado-descongelado. Comparación de dos métodos. Archivos de Medicina Veterinaria, 1994; 26: 25-40.

(20) Urrego R., Ríos A., Olivera M., Camargo O. Efecto de la centrifugación sobre la membrana plasmática y el ADN de espermatozoides bovinos. Revista Colombiana de Ciencias Pecuarias, 2008; 21: 19-26.

(21) Pérez-Llano B., Lorenzo JL, Yenes P., Trejo A., García-Casado P. A short hypoosmotic swelling test for the prediction of boar sperm fertility. Theriogenology, 2001, 56: 387398. DOI: http://dx.doi.org/10.1016/S0093691X(01)00571-4.

(22) Prathalingam NS, Watson PF, Revell SG, Busby J., Holt WV. The response of bovine spermatozoa to bicarbonate an its use to assess the influence of added oviductal epithelial proteins on cryopreservation. Journal of Andrology, 2007; 28: 407-415. DOI: http:// dx.doi.org/10.2164/jandrol.106.001594.

(23) Revell SG, Mrode RA. An osmotic resistance test for bovine semen. Animal Reproduction Science, 1994; 36: 77-86. DOI: http://dx.doi. org/10.1016/0378-4320(94)90055-8.

(24) Rubio L., Quintero AA, González DM. Efecto de la criopreservación sobre la integridad de la membrana plasmática y acrosomal de espermatozoides de toros. Revista Científica, FCV-LUZ, 2009; 19: 382-389.

(25) Díaz O., Mesa H., Gómez G., Henao FJ. Evaluación invitro de la viabilidad del semen porcino hasta 120 horas de almacenamiento en refrigeración. Veterinaria y Zootecnia, 2009; 3: 32-37.
(26) Catena M., Cabodevila J. Evaluación del semen congelado. Taurus, 1999; 1: 18-31.

(27) Grub M., Wattiaux M. Curso de inseminación artificial. 2006.

(28) Looper M. Proper semen handling iomproves conception rates of dairy cows. Extension Dairy Specialist Guide 2000; D: 1-4.

(29) Drobnis E. et al. Cold shock damage is due to lipid phase transitions in cell membranes: A demostration using sperm as a model. J Exp Zool, 1993; 19: 432-437. DOI: http://dx.doi. org/10.1002/jez.1402650413.

(30) Januskauskas A., Johannisson A., Soderquist L., Rodríguez-Martínez $\mathrm{H}$. Assessment of sperm characteristics post-thaw and responde to calcium ionophore in relation to fertility in Swedish dairy Al bulls. Theriogenol, 2000; 53: 859-875. DOI: http://dx.doi.org/10.1016/ S0093-691X(00)00235-1.

(31) Cabrera P., Pantoja A. Viabilidad espermática e integridad del acrosoma en semen congelado de toros nacionales. REV Inv Vet Perú, 2012; 23(2): 192-200. DOI: http://dx.doi. org/10.15381/rivep.v23i2.899.

(32) Senger PL. Handling frozen bovine semen-factors wick influence viability and fertility. Theriogenology, 1980; 13:51.62.

(33) Santamaría A. Factores que influyen sobre la fertilidad y viabilidad del semen bovino congelado. Monografías de Medicina Veterinaria, $1988 ; 10$.

(34) Pace MM, Sullivan JJ. A biological comparison of the $0.5 \mathrm{ml}$ ampute and $0.5 \mathrm{ml}$ French straw system for packaging bovine spermatozoa. PROC. 7th. Tech. Conf. Artif. Insem. Reprod. $1978 ; 22-23$.

(35) Lineweaver JA, Saacke RG, Gwasdauskas FC. Effect of double decking and storage time on semen stored in farm storage tanks (Abstr) 
Prod. 1979 American Dairy Sci. Assoc, 1980; 175.

(36) Villa N. Evaluación del efecto de la Técnica Instrumental sobre la calidad del semen bovino, congelado-descongelado, en el Centro de Colombia. Trabajo de Maestría. Universidad de Caldas. Facultad de Medicina Veterinaria y Zootecnia. Manizales. Colombia, 2010.

(37) Bernardi SF, Allende R., Mazzeo R., Monti J., Marini PR. Evaluación de los cambios ocasionados en espermatozoides bovinos en el manejo de las dosis durante su manipulación en inseminación artificial. In. Vet., 2011; 13(2): 25-38.

(38) Rubio-Guillén J., González D., González, Madrid N., Quintero A. ¿Puede el ORT complementar las pruebas clásicas de valoración seminal y predecir la fertilidad en toros? Arch Latinoam Prod Anim, 2007; 15(1): 329-333.

(39) Estevez S., Sharma R., Thomas A., Agarwal A. Improvement in motion characteristic and acrosome status in cryopreserved human spermatozoa by swim-up processing before freezing. Human Reprod, 2000; 15 (10):
2173-2179. DOI: http://dx.doi.org/10.1093/ humrep/15.10.2173.

(40) Quintero AA. Estudio sobre la dinámica de poblaciones espermáticas en semen de cabaIlo, cerdo y conejo. Tesis de doctorado, Universidad Autónoma de Barcelona, Bellaterra, 2003.

(41) Bollwein, H.; Fuchs, I.; Koess, C. Interrelationship Between Plasma Membrane Integrity, Mitochondrial Membrane Potential and DNA Fragmentation in Cryopreserved Bovine Spermatozoa. Reproduction Domestic Animal, 2008; 43(2): 189-195. DOI: http://dx.doi.org/10.1111/j.1439-0531.2007.00876.x.

(42) Thundathil J., Gil J. et al. Relationship between the proportion of capacitated spermatozoa present in frozen-thawed bull semen and fertility with artificial insemination. Int J Androl, 1999; 22 (6): 366-73. DOI: http://dx.doi. org/10.1046/j.1365-2605.1999.00194.x.

(43) Roncoletta M., Morani Eda S. et al. Fertility associated proteins in Nelore bull sperm membranes. Animal Reproduction Science, 2006; 91(1-2):77-87. DOI: http://dx.doi.org/10.1016/j.anireprosci.2005.03.014. 